\title{
Fuzzy Preferences Graph Model to Study a Water Pollution Conflict
}

\author{
Jing $\mathrm{Yu}^{1,2,3, *}$, Jackson $\mathrm{Chen}^{4}$ and Min Zhao ${ }^{5,6}$
}

${ }^{1}$ School of Economics and Management, Nanjing University of Science and Technology, Nanjing, Jiangsu, 210094
China; ${ }^{2}$ Research Center for International Economy and Trade, Nanjing University of Science and Technology,
Nanjing, Jiangsu, 210094 China; ${ }^{3}$ Research Center for Low-carbon Economy and Environmental Policy, Nanjing
University of Science and Technology, Nanjing, Jiangsu, 210094 China; ${ }^{4}$ Fairview High School, Boulder, Colorado
80305 , USA; ${ }^{5}$ Business School, Hohai University, Nanjing 211100 , P.R. China; ${ }^{6}$ Water Resources and Sustainable De-
velopment Research Center of Jiangsu Province, Nanjing 211100, P.R. China

Abstract: The fuzzy preference framework of Graph Model for Conflict Resolution (GMCR), handling both crisp and fuzzy preferences of decision makers (DMs), is capable of calculating and predicting different conflict resolutions (equilibria) according to DMs' different satisficing criteria. Employing this methodology, a water contamination conflict occurs at the boundary of Jiangsu and Zhejiang provinces in China is investigated to provide strategic insights for both practitioners and researchers of the conflict. Additionally, a useful tool called fuzzy option prioritization, which can efficiently represent both crisp and fuzzy preferences of DMs, is introduced briefly and applied to elicit DMs' preferences in the modeling process of the water pollution dispute. The analysis results demonstrate the applicability of the fuzzy preference framework of GMCR for systematically modeling and analyzing real-world water resources conflicts, and the usefulness of the fuzzy option prioritization technique for simplifying the process of obtaining DMs' fuzzy preferences.

Keywords: Conflict resolution, fuzzy equilibrium, fuzzy preferences, graph model, option prioritization, water resources conflict

\section{INTRODUCTION}

Water resources conflict has become a global wide phenomenon with the population growth and the development of social economy. One can easily find various water resources disputes arise at both regional and international level. Generally, water resources conflicts are highly complex strategic decision problems and are mainly caused by the different needs for water quantity or water quality among different stakeholders. The increasing water resources disputes seriously restrict the sustainable utilization of the limited water resources. Therefore, various quantitative and qualitative conflict resolution methods have been developed for water resources management. Among these methods, the Graph Model for Conflict Resolution (GMCR) [1, 2] has been demonstrated to be a useful qualitative tool for systematically modeling and analyzing strategic conflicts, because it can show a better understanding of water resources conflicts and can illustrate how to effectively manage water resources in more strategic and positive ways.

As a comprehensive, simple, and flexible methodology, GMCR holds many advantages. For instance, it needs only decision makers' (DMs) relative preferences. It is capable of modeling both irreversible and common moves. It provides a flexible framework to define and compare different stability concepts and is easy to apply to real-world conflicts. Therefore, GMCR has been employed to analyze and solve conflicts in various fields, including water resources management, brownfield redevelopment, environmental protection and other types of disputes [3-5].

\footnotetext{
*Address correspondence to this author at the School of Economics and Management of Nanjing University of Science and Technology, Nanjing, 210094, P.R. China; Tel: 025-68514713; E-mail: yujing_1226@126.com
}

Using GMCR to investigate a strategic conflict contains two steps: firstly, modeling or representing a real-world conflict within a formal framework; secondly, conducting stability analysis and other subsequent analysis, such as coalition analysis [6], status quo analysis [7], and attitude analysis [8, 9] if necessary [10]. Relative preferences information for each DM over all feasible states, is one of the most important inputs in the modeling process of GMCR, and will have significant influence on the stability analysis results and other subsequent analysis results. Generally, the relative preferences in GMCR are represented by a crisp preference relation $\gtrsim$, where $>$ indicates "is (strictly) preferred to" and means "is indifferent to" [2]. A crisp preference describes the certainty of the preference for one state over another.

However, in real-world contexts, DMs are often uncertain or unclear about preferences between two states due to the reasons of lack of information, cultural or educational factors, the inherent vagueness of human judgment, or the conflict is ongoing. Hence, Bashar et al. [11] formally introduced fuzzy preferences into the framework of GMCR to determine the strategic impacts of preference uncertainty. A fuzzy preference relation is a pairwise preference degree expressed using numerical values between 0 and 1 . Specifically, a preference degree for one state over another interprets the degree of certainty of the preference for the first state over the second. A degree of certainty of 1 is equivalent to the preference relation "strictly preferred to", while a degree of certainty of 0 is equivalent to equal certainty but in the reverse direction.

Since expressing preference by pair-wise comparison is a challenging task for both DMs and analysts, especially when the conflict model possesses a large number of feasible 
states, an efficient and easy technique, crisp option prioritization, was developed in GMCR II [12-14] to facilitate the process of eliciting DMs' crisp preferences. Based on this method, Bashar et al. [15] proposed a fuzzy option prioritization methodology, which is employed to elicit fuzzy preferences in this paper. Until now a little work has been done to express fuzzy preferences using the novel fuzzy option prioritization technique, and to study real-world water resources conflicts using GMCR with DMs' fuzzy preferences. In this paper, the fuzzy preference framework of GMCR and the fuzzy option prioritization method are utilized to model and analyze a water contamination conflict which occurs in China to obtain more strategic insights. In the next section, the structure of GMCR incorporating fuzzy preferences and the fuzzy option prioritization approach are described briefly, followed by the application of these methodologies to investigate the water pollution dispute. Finally, some conclusions are drawn and some directions for future work are given.

\section{METHODS}

\subsection{GMCR under Fuzzy Preferences}

A fuzzy preference framework of GMCR is usually represented by $G=\left\langle N, S,\left\{A_{i}\right\}_{i \in N},\left\{\mathcal{R}_{i}\right\}_{i \in N}\right\rangle$, where $N=$ $\{1,2, \ldots, i, \ldots, n-1, n\}$ is a set of DMs; $S=\left\{s_{1}, s_{2}, \cdots\right.$, $\left.s_{k}, \cdots, s_{t}, \cdots, s_{w}\right\}$ is a set of feasible states; $\left\{\mathcal{R}_{i}\right\}_{i \in N}$ represents a set of fuzzy preference relationships on $S$ for all DMs, where $\mathcal{R}_{i}$ indicates DM $i$ 's fuzzy preference matrix; and $A_{i} \subseteq S \times S$ is a set of oriented arcs, together with node set $S$, an integrated and directed graph for a conflict can be drawn as $\left\langle S,\left\{A_{i}\right\}_{i \in N}\right\rangle$.

A fuzzy preference of DM $i$ over $S$ is a fuzzy relation on $\mathrm{S}$, represented by a matrix $\mathcal{R}_{i}=\left(r_{k t}\right)_{w \times w}$, with membership function: $\mu_{\mathcal{R}}: S \times S \rightarrow[0,1]$, where $\mu_{\mathcal{R}}\left(s_{k}, s_{t}\right)=r_{k t}$ denotes the preference degree of state $s_{k}$ over $s_{t}$, satisfying $r_{k t}+r_{t k}=1$ and $r_{k k}=0.5$. Note that the preference degree is interpreted as the level of certainty that a DM will prefer one state to the other but says nothing about how great this preference is likely to be.

The concept of fuzzy relative strength of preference (FRSP) is given by Bashar et al. [11] to measure how strongly a DM prefers one state to the other. Specifically, let $r_{k t}^{i}$ denote the preference degree of state $s_{k}$ over $s_{t}$ for DM $i$. Then DM $i$ 's FRSP of state $s_{k}$ over $s_{t}$ is defined to be $\alpha^{i}\left(s_{k}, s_{t}\right)=r_{k t}^{i}-r_{t k}^{i}$, where $-1 \leq \alpha^{i}\left(s_{k}, s_{t}\right) \leq 1$.

For the stability analysis in GMCR, one important task is to determine whether a DM is better to stay at a focal state or to move to other states. Every DM with fuzzy preferences in a conflict model may select a level of FRSP to justify whether a move from one state to another is worthwhile. This level of FRSP is referred to as the DM's fuzzy satisficing threshold (FST), which is formally defined as: for $i \in N$, DM $i$ 's FST is denoted by $\gamma_{i}$, then DM $i$ would be willing to move from state $s_{t}$ to state $s_{k}$ if and only if $\alpha^{i}\left(s_{k}, s_{t}\right) \geq \gamma_{i}$, where $0<\gamma_{i} \leq 1$.
FST is a parameter that reflects a DM's criterion in identifying a move to a state that is sufficiently likely to be preferable. Note that different DMs in a conflict may hold different FSTs to determine their own fuzzy stable states; one specific DM may have different FTSs at different time or under different situations.

With the above concepts, four fuzzy stability definitions (FNash, FGMR, FSMR and FSEQ) are proposed by Bashar et al. [11] to calculate the possible solutions or equilibria of a conflict. See [11] for details about the four fuzzy stability definitions.

\subsection{Fuzzy Option Prioritization}

The basic idea of the crisp option prioritization approach is to let a DM provide a priority sequence of preference statements describing his or her preferences about the available options or courses of actions, and then assign a truth value ("true" or "false") to each preference statement at each state. A DM's crisp preference between two states is determined based on the truth values of the preference statements at all states in lexicographic ordering fashion.

Bashar et al. [15] extended the crisp option prioritization method to model both crisp and fuzzy preferences through using fuzzy truth values (numerical values between 0 and 1) of preference statements instead of truth values in the crisp option prioritization. A fuzzy truth degree of 1 is equivalent to 100 percent of truth in the crisp option prioritization, while a fuzzy truth degree of 0 is equivalent to 100 percent of false. Specifically, the new methodology is to generate a fuzzy score interval for each feasible state based on the fuzzy truth values of preference statements at each state. A fuzzy preference degree for one state over another can then be calculated by comparing the fuzzy score intervals of the two states.

\section{CASE STUDY}

Maxi Port is a small river located at the boundary of Shengze Town (in Suzhou City, Jiangsu Province) and Wangjiangjing Town (in Jiaxing City, Zhejiang Province) in China. The river flows from Shengze Town to the northern part of Jiaxing City. The 27 big dyeing and printing enterprises in Shengze Town released large quantities of untreated industrial wastewater into Maxi Port, which caused serious water pollution in the northern areas of Jiaxing City. In 1990s, disputes frequently arose at the border of Jiangsu and Zhejiang because the pollution was never properly solved. Eventually, on 22 November 2001, to block industrial wastewater released into the Maxi River by factories in Shengze Town from reaching the northern part of Jiaxing City, citizens from Jiaxing who self-raised over one million yuan, sank 28 concrete boats at Maxi Port. This event attracted great attention of the Central Government (CG) of China. Finally, through the CG's coordination, Jiangsu and Zhejiang conducted a formal negotiation which resulted in an agreement. Under this agreement, Suzhou had to reduce its pollution emissions to less than $40 \%$ of the original, and subsequently, Zhejiang removed the blockage. See [16] for more details about this conflict. 
Table 1. DMs, options and feasible states.

\begin{tabular}{|c|c|c|c|c|c|c|c|c|c|c|c|c|c|c|}
\hline DMs & Options & $s_{1}$ & $s_{2}$ & $s_{3}$ & $s_{4}$ & $s_{5}$ & $s_{6}$ & $s_{7}$ & $s_{8}$ & $s_{9}$ & $s_{10}$ & $s_{11}$ & $s_{12}$ & $s_{13}$ \\
\hline \multirow{3}{*}{ JS } & $\mathrm{A}_{1}$ : Retain & $\mathrm{Y}$ & $\mathrm{Y}$ & $\mathrm{N}$ & $\mathrm{N}$ & $\mathrm{Y}$ & $\mathrm{Y}$ & $\mathrm{N}$ & $\mathrm{N}$ & $\mathrm{Y}$ & $\mathrm{Y}$ & $\mathrm{N}$ & $\mathrm{N}$ & - \\
\hline & $\mathrm{A}_{2}$ : Reduce & $\mathrm{N}$ & $\mathrm{N}$ & $\mathrm{Y}$ & $\mathrm{Y}$ & $\mathrm{N}$ & $\mathrm{N}$ & $\mathrm{Y}$ & $\mathrm{Y}$ & $\mathrm{N}$ & $\mathrm{N}$ & $\mathrm{Y}$ & $\mathrm{Y}$ & - \\
\hline & $\mathrm{A}_{3}:$ Close & $\mathrm{N}$ & $\mathrm{N}$ & $\mathrm{N}$ & $\mathrm{N}$ & $\mathrm{N}$ & $\mathrm{N}$ & $\mathrm{N}$ & $\mathrm{N}$ & $\mathrm{N}$ & $\mathrm{N}$ & $\mathrm{N}$ & $\mathrm{N}$ & $\mathrm{Y}$ \\
\hline \multirow{2}{*}{ ZJ } & $\mathrm{B}_{1}$ : Negotiate & $\mathrm{Y}$ & $\mathrm{N}$ & $\mathrm{Y}$ & $\mathrm{N}$ & $\mathrm{Y}$ & $\mathrm{N}$ & $\mathrm{Y}$ & $\mathrm{N}$ & $\mathrm{Y}$ & $\mathrm{N}$ & $\mathrm{Y}$ & $\mathrm{N}$ & - \\
\hline & $\mathrm{B}_{2}:$ Block & $\mathrm{N}$ & Y & $\mathrm{N}$ & Y & $\mathrm{N}$ & Y & $\mathrm{N}$ & Y & $\mathrm{N}$ & $\mathrm{Y}$ & $\mathrm{N}$ & $\mathrm{Y}$ & - \\
\hline \multirow{3}{*}{$\mathrm{CG}$} & $\mathrm{C}_{1}$ : Persuade & $\mathrm{Y}$ & $\mathrm{Y}$ & $\mathrm{Y}$ & $\mathrm{Y}$ & $\mathrm{N}$ & $\mathrm{N}$ & $\mathrm{N}$ & $\mathrm{N}$ & $\mathrm{N}$ & $\mathrm{N}$ & $\mathrm{N}$ & $\mathrm{N}$ & - \\
\hline & $\mathrm{C}_{2}$ : Reward & $\mathrm{N}$ & $\mathrm{N}$ & $\mathrm{N}$ & $\mathrm{N}$ & $\mathrm{Y}$ & $\mathrm{Y}$ & $\mathrm{Y}$ & $\mathrm{Y}$ & $\mathrm{N}$ & $\mathrm{N}$ & $\mathrm{N}$ & $\mathrm{N}$ & - \\
\hline & $\mathrm{C}_{3}$ : Punish & $\mathrm{N}$ & $\mathrm{N}$ & $\mathrm{N}$ & $\mathrm{N}$ & $\mathrm{N}$ & $\mathrm{N}$ & $\mathrm{N}$ & $\mathrm{N}$ & $\mathrm{Y}$ & $\mathrm{Y}$ & $\mathrm{Y}$ & $\mathrm{Y}$ & - \\
\hline
\end{tabular}

\subsection{Conflict Modeling}

\subsubsection{DMs, Options and Feasible States}

As Suzhou City and Jiaxing City individually fall under the control of Jiangsu (JS) Province and Zhejiang (ZJ) Province, respectively, the DMs in the water pollution conflict are the upstream region located in JS, the downstream region in $\mathrm{ZJ}$, and $\mathrm{CG}$, which is the coordinator. JS possesses three available options under its control: $\mathrm{A}_{1}$ : Retain-Retain the status quo by keeping the existing economic growth mode and doing nothing about the pollution; $\mathrm{A}_{2}$ : Reduce-Reduce the pollution by decreasing wastewater emissions; $A_{3}$ : Close-Shut down the polluting industries to completely eliminate their emissions. ZJ has two options: $\mathrm{B}_{1}$ : NegotiateTry to make JS reduce its pollution through negotiation; $\mathrm{B}_{2}$ : Block-Try to force JS to reduce its pollution via coercive action. There are three options for CG: $\mathrm{C}_{1}$ : PersuadePersuade JS to reduce its pollution; $\mathrm{C}_{2}$ : Reward-Encourage JS to reduce its pollution by providing capital or technological support to JS; $\mathrm{C}_{3}$ : Punish-Force JS to reduce the pollution through setting water quality standards in certain sections of the river, and punish JS if the water quality is substandard.

From a logical point of view, the conflict among three DMs, with a total of eight options will produce $2^{8}=256$ states. However, 13 feasible states (labeled as $s_{1}, s_{2}, \ldots, s_{13}$ ) finally remain as shown in Table $\mathbf{1}$ after the infeasible ones are eliminated. For instance, each DM must choose at least and at most one option at one time. State $s_{13}$ is an indistinguishable state since the conflict is solved no matter what $\mathrm{ZJ}$ and CG react as long as JS chooses option $\mathrm{A}_{3}$. In Table 1, "Y" means the option is selected by the DM controlling it; "N" indicates that it is not taken; while a dash ("“-) stands for $\mathrm{Y}$ or $\mathrm{N}$.

Fig. (1) shows the integrated graph model of the water pollution conflict, where the feasible states are indicated by the circles, while the state transitions controlled by different DMs are represented by the directed arcs drawn in different types of lines. There are tails that represent the initial states and the arrowheads represent the terminal states moved from the initial states. For example, CG can cause the conflict to move from state $s_{1}$ to state $s_{9}$ by changing its option selection from "Persuade" to "Punish," as indicated in states $s_{1}$ and $s_{9}$ in Table 1 for which the option selections of the other two DMs remain fixed, and similarly, CG can also cause the conflict to move from state $s_{9}$ to state $s_{1}$ by changing its option selection from "Punish" to "Persuade." Note that the state transition might or might not be reversible. The dashed box with one-way arrowhead, for instance, means that JS can move from any of the 12 feasible states inside the dashed box to state $s_{13}$, but JS cannot move from state $s_{13}$ to any of the 12 feasible states inside the dashed box.

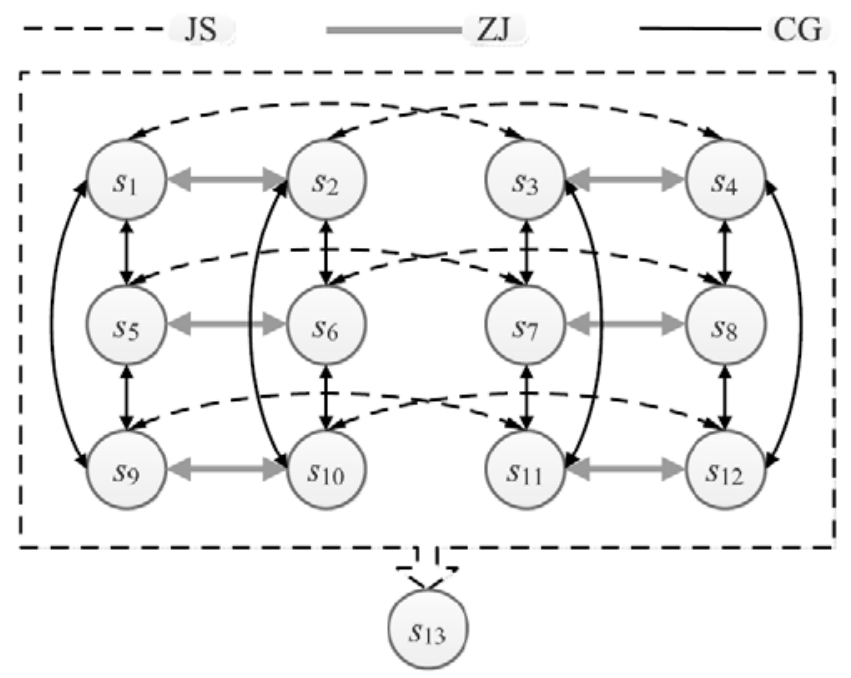

Fig. (1). The integrated graph model.

\subsubsection{Fuzzy Preferences}

Option prioritization furnishes an intuitive specification based on prioritized preference statements composed in terms of options and logical connectives. Tables $\mathbf{2}$ to $\mathbf{4}$ contain explanations of the preference statements listed from the most important at the top to least important at the bottom for JS, JZ and CG, respectively. For example, as shown at the top of Table $\mathbf{2}$ on the left column, the most important preference statement for JS is that do not choose "Close" to completely reduce the pollution, which is indicated by a negative sign beside $\mathrm{A}_{3}$. Notice that a DM can use conditional preference statements, such like the third important preference statement $\left(\mathrm{A}_{1} \mathrm{IF} \mathrm{C}_{1}\right)$ for JS, with the explanation that JS prefers to choose "Retain" if CG selects "Persuade". 
Table 2. JS's preference statements.

\begin{tabular}{|c|c|}
\hline Statements & Descriptions \\
\hline \hline$-\mathrm{A}_{3}$ & Do not choose "Close" to completely reduce the pollution \\
\hline $\mathrm{C}_{1}$ & $\mathrm{CG}$ selects "Persuade" to coordinate the conflict \\
\hline $\mathrm{A}_{1}$ IF C & Choose "Retain" if CG selects "Persuade" \\
\hline $\mathrm{A}_{2}$ IF $\mathrm{C}_{2} \mid \mathrm{C}_{3}$ & Choose "Reduce" if CG selects "Reward" or "Punish" \\
\hline $\mathrm{C}_{2}$ & CG selects "Reward" to coordinate the conflict \\
\hline$-\mathrm{B}_{2}$ & ZJ does not select "Block" to upgrade the conflict \\
\hline
\end{tabular}

Table 3. ZJ's preference statements.

\begin{tabular}{|c|c|}
\hline Statements & Descriptions \\
\hline \hline $\mathrm{A}_{3}$ & JS chooses "Close" to completely reduce the pollution \\
\hline $\mathrm{A}_{2}$ & JS selects "Reduce" to partially reduce the pollution \\
\hline $\mathrm{B}_{1}$ IF $\mathrm{A}_{2}$ & $\begin{array}{r}\text { Choose "Negotiate" to cooperate with JS if JS chooses } \\
\text { "Reduce" to partially reduce the pollution }\end{array}$ \\
\hline $\mathrm{B}_{2} \mathrm{IF} \mathrm{A}_{1}$ & $\begin{array}{r}\text { Select "Block" to intensify the conflict if JS chooses } \\
\text { "Retain" to do nothing about the pollution }\end{array}$ \\
\hline $\mathrm{C}_{3}$ & $\mathrm{CG}$ chooses "Punish" to coordinate the conflict \\
\hline $\mathrm{C}_{2}$ & $\mathrm{CG}$ selects "Reward" to coordinate the conflict \\
\hline
\end{tabular}

JS's preferences over some states are uncertain, and the uncertainty can be reflected from JS's fuzzy judgments of the truth values of certain preference statements at particular states. For instance, generally, the truth value of JS's preference statement "- $\mathrm{A}_{3}$ " (meaning that JS prefers not to close its polluting industries) is "true" at states $s_{10}$ and $s_{12}$, and is "false" at state $s_{13}$. However, when $\mathrm{ZJ}$ holds noncooperative attitude and CG carries out punitive policy, suf-
Table 4. CG's preference statements.

\begin{tabular}{|c|c|}
\hline Statements & Descriptions \\
\hline \hline $\mathrm{A}_{3}$ & JS chooses "Close" to completely reduce the pollution \\
\hline $\mathrm{A}_{2}$ & JS selects "Reduce" to partially reduce the pollution \\
\hline$-\mathrm{B}_{2}$ & ZJ does not choose "Block" to escalate the conflict \\
\hline $\mathrm{C}_{3}$ & Select "Punish" to coordinate the conflict \\
\hline $\mathrm{C}_{2}$ & Choose "Reward" to coordinate the conflict \\
\hline
\end{tabular}

fering all these pressures, JS might consider that state $s_{13}$ is better than states $s_{10}$ and $s_{12}$. Thus, JS may not prefer to choose "not close" with $100 \%$ truth (i.e. a truth degree of 1); instead, JS may prefer to choose "not close" with certain degree of truth between 0 and 1 . In this case, the truth values of JS's preference statement "- $\mathrm{A}_{3}$ " at states $s_{10}, s_{12}$, and $s_{13}$ might be $0.8,0.6$, and 0.1 , respectively. A similar argument may be given for JS when judging the truthfulness of the preference statement " $\mathrm{C}_{1}$ " (meaning that JS prefers that CG selects "Persuade" to coordinate the conflict) at states $S_{3}, s_{4}$, $s_{7}$, and $s_{8}$, because when JS chooses "Reduce", JS might want some policy compensation from CG (i.e. may prefer CG choose "Reward" instead of "Persuade"). Specifically, JS might assign a non-zero truth degree to " $\mathrm{C}_{1}$ " at states $S_{7}$ and $s_{8}$ rather than a truth value of "false" (equivalent to a truth degree of 0 ), while assign a non-one truth degree to " $\mathrm{C}_{1}$ " at states $s_{3}$ and $s_{4}$ rather than a truth value of "truth" (equivalent to a truth degree of 1). Thus, the truth values of JS's preference statement " $\mathrm{C}_{1}$ " at states $s_{3}, s_{4}, s_{7}$, and $s_{8}$ are assumed to be $0.35,0.3,0.45$, and 0.55 , respectively in this paper. Taking the above and similar circumstances into account, the fuzzy truth values of JS's preference statements at all states are assigned as shown in the second column of Table 5 .

Table 5. Fuzzy truth values.

\begin{tabular}{|c|c|c|c|}
\hline \multirow{2}{*}{ States } & \multicolumn{2}{|c|}{ Fuzzy Truth Values of Preference Statements (Most Important to Least) at State } \\
\cline { 2 - 4 } & JS & ZJ & $(0,0,1,0,0)$ \\
\hline \hline$s_{1}$ & $(1,1,1,1,0,1)$ & $(0,0,1,0,0,0)$ & $(0,0,0,0,0)$ \\
\hline$s_{2}$ & $(1,1,0.6,1,0,0)$ & $(0,0,1,1,0,0)$ & $(0,1,1,0,0)$ \\
\hline$s_{3}$ & $(1,0.35,0,1,0,1)$ & $(0,1,1,1,0,0)$ & $(0,1,0,0,0)$ \\
\hline$s_{4}$ & $(1,0.3,0.2,1,0,0)$ & $(0,1,0,1,0,0)$ & $(0,0,1,0,1)$ \\
\hline$s_{5}$ & $(1,0,1,0.15,1,1)$ & $(0,0,1,0,0,1)$ & $(0,0,0,0,1)$ \\
\hline$s_{6}$ & $(1,0,1,0,1,0)$ & $(0,0,1,1,0,1)$ & $(0,1,1,0,1)$ \\
\hline$s_{7}$ & $(1,0.45,1,0.65,1,1)$ & $(0,1,1,1,0,1)$ & $(0,1,0,0,1)$ \\
\hline$s_{8}$ & $(1,0.55,1,1,1,0)$ & $(0,1,0,1,0,1)$ & $(0,0,1,1,0)$ \\
\hline$s_{9}$ & $(1,0,1,0,0,1)$ & $(0,0,1,0,1,0)$ & $(0,0,0,1,0)$ \\
\hline$s_{10}$ & $(0.8,0,1,0,0,0)$ & $(0,0,1,1,1,0)$ & $(0,1,1,1,0)$ \\
\hline$s_{11}$ & $(1,0,1,1,0,1)$ & $(0,1,1,1,1,0)$ & $(0,1,0,1,0)$ \\
\hline$s_{13}$ & $(0.6,0,1,1,0,0)$ & $(0,1,0,1,1,0)$ & $(1,0,1,0,0)$ \\
\hline
\end{tabular}


Table 6. The fuzzy preferences of JS.

\begin{tabular}{|c|c|c|c|c|c|c|c|c|c|c|c|c|c|c|}
\hline & & $s$ & 1 & $s_{3}$ & $S_{4}$ & $S_{5}$ & $s_{6}$ & $S_{7}$ & $s_{8}$ & $s_{9}$ & $s_{10}$ & \multicolumn{3}{|c|}{$s_{12} \quad s_{13}$} \\
\hline & $s_{1}$ & 0.5 & 1.0 & 1.0 & 1.0 & 1.0 & 1.0 & 1.0 & 1.0 & 1.0 & 1.0 & 1.0 & \multicolumn{2}{|c|}{ 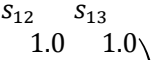 } \\
\hline & $s_{2}$ & 0 & 0.5 & 1.0 & 1.0 & 1.0 & 1.0 & 0.8 & 0.67 & 1.0 & 1.0 & 1.0 & 1.0 & 1.0 \\
\hline & $s_{3}$ & 0 & 0 & 0.5 & 0.51 & 0.38 & 0.58 & 0 & 0 & 0.72 & 1 & 0.17 & 1.0 & 1.0 \\
\hline & $S_{4}$ & 0 & 0 & 0.49 & 0.5 & 0.38 & 0.54 & 0 & 0 & 0.65 & 0.95 & 0.22 & 0.95 & 1.0 \\
\hline & $S_{5}$ & 0 & 0 & 0.62 & 0.62 & 0.5 & 1.0 & 0 & 0 & 1.0 & 1.0 & 0 & 1.0 & 1.0 \\
\hline & $s_{6}$ & 0 & 0 & 0.42 & 0.46 & 0 & 0.5 & 0 & 0 & 1.0 & 1.0 & 0 & 1.0 & 1.0 \\
\hline & $s_{7}$ & 0 & 0.2 & 1.0 & 1.0 & 1.0 & 1.0 & 0.5 & 0.39 & 1.0 & 1.0 & 1.0 & 1.0 & 1.0 \\
\hline & $s_{8}$ & 0 & 0.33 & 1.0 & 1.0 & 1.0 & 1.0 & 0.61 & 0.5 & 1.0 & 1.0 & 1.0 & 1.0 & 1.0 \\
\hline & $s_{9}$ & 0 & 0 & 0.28 & 0.35 & 0 & 0 & 0 & 0 & 0.5 & 1.0 & 0 & 1.0 & 1.0 \\
\hline & $s_{10}$ & 0 & 0 & 0 & 0.05 & 0 & 0 & 0 & 0 & 0 & 0.5 & 0 & 0.6 & 1.0 \\
\hline & $s_{11}$ & 0 & 0 & 0.83 & 0.78 & 1.0 & 1.0 & 0 & 0 & 1.0 & 1.0 & 0.5 & 1.0 & 1.0 \\
\hline & $s_{12}$ & 0 & 0 & 0 & 0.05 & 0 & 0 & 0 & 0 & 0 & 0.4 & 0 & 0.5 & 1.0 \\
\hline & $s_{13}$ & 10 & 0 & 0 & 0 & 0 & 0 & 0 & 0 & 0 & 0 & 0 & 0 & 0.5 \\
\hline
\end{tabular}

Table 7. The crisp preferences of $\mathrm{ZJ}$ and $\mathbf{C G}$.

\begin{tabular}{|c|c|}
\hline DMs & Preference Rankings \\
\hline \hline ZJ & $s_{13}>s_{11}>s_{7}>s_{3}>s_{12}>s_{8}>s_{4}>s_{10}>s_{6}>s_{2}>s_{9}>s_{5}>s_{1}$ \\
\hline CG & $s_{13}>s_{11}>s_{7}>s_{3}>s_{12}>s_{8}>s_{4}>s_{9}>s_{5}>s_{1}>s_{10}>s_{6}>s_{2}$ \\
\hline
\end{tabular}

As the objective of both $\mathrm{ZJ}$ and $\mathrm{CG}$ is reducing the pollution, they may therefore have more precisely defined preferences. Accordingly, the truth value of each preference statement of ZJ and CG at each state is either "true" or "false", i.e. is either " 1 " or " 0 ", as presented in the third and fourth columns of Table $\mathbf{5}$.

In Tables 2, 3 and 4, there are a total of 6, 6 and 5 preference statements for JS, ZJ and CG, respectively. Since there is exactly one truth degree for one preference statement at a given state, JS, ZJ and CG have 6, 6 and 5 truth degrees, respectively, at each state. The second, third and fourth columns of Table 5 show these truth degrees as 6-tuples, 6tuples and 5-tuples, respectively, in which the truth degrees appear in the decreasing order of importance of the preference statements (from most important on the left to least important on the right). For example, in the 6-tuple $(1,0.35,0,1,0,1)$ in the third row and second column of Table 5, the first entry 1 is the truth degree of the most important preference statement "- $\mathrm{A}_{3}$ " of JS at state $s_{3}$, the second entry 0.35 is the truth degree of the second most important preference statement " $\mathrm{C}_{1}$ ", and so on.

With the fuzzy truth values in Table 5, the fuzzy preference degrees for JS, ZJ and CG can be calculated by employing the fuzzy option prioritization technique. From the results, it is clear that the preferences of JS, represented by the matrix $\mathcal{R}_{J S}$ in Table 6, are fuzzy. In the matrix, each entry represents the degree of preference for a row state over a column state. However, the preferences of $\mathrm{ZJ}$ and $\mathrm{CG}$, as shown from most preferred on the left to least preferred on the right in Table 7, are crisp, because the truth degrees of the preference statements of ZJ and CG at feasible states are either 1 or 0 .

\subsection{Conflict Analysis}

\subsubsection{Fuzzy Stability Analysis}

When employing the four fuzzy stability definitions (FNash, FGMR, FSMR, FSEQ) to carry out a fuzzy stability analysis, DMs' FTSs usually play a vital role. To demonstrate how the satisficing behavior of JS influences its preferences and the fuzzy stabilities of the conflict, two different FSTs of JS are considered. The FSTs of JS used in the analysis in this paper are 1) $\gamma_{1}=0.2$;2) $\gamma_{1}=0.5$. As ZJ and CG have crisp preferences, they posses equal FST of 1, i.e. $\gamma_{2}=\gamma_{3}=1.0$. The fuzzy stability analysis results are presented in Table $\mathbf{8}$, where " $\Delta$ " in a cell indicates that the state in the corresponding row is fuzzy stable for the particular DM under the given fuzzy stability concept; "๑" in a cell indicates that the state is fuzzy stable for all DMs, and thus is a fuzzy equilibrium (FE) of the conflict under the given fuzzy stability definition. A state is a fuzzy stable state for a $\mathrm{DM}$, if and only if under the given fuzzy stability definition, the DM's FRSP of moving from the focal state to any other states is less than its FST. A state constitutes a FE of the conflict if the state is fuzzy stable for all DMs.

As can be seen from Table $\mathbf{8}$, when weaker satisficing criteria for JS $\left(\gamma_{1}=0.2\right)$ is considered, JS is more likely to prefer to choose "Retain" than "Reduce" (i.e. JS is more economic-oriented), which results in that state $s_{10}$ is more preferred by JS than state $s_{12}$, and states $s_{10}, s_{11}, s_{13}$ are strongly fuzzy stable states (fuzzy stable for all DMs under all the four fuzzy stability concepts), while states $s_{3}, s_{4}, s_{7}$, $S_{8}$ are weakly fuzzy stable states (only satisfy FGMR and FSMR stabilities). However, for stronger satisficing criteria for JS $\left(\gamma_{1}=0.5\right)$, that is, for increased FST, JS is more likely to prefer to choose "Reduce" than "Retain" (i.e. JS is more environmental-oriented), which results in that state $s_{12}$ is more preferred by JS than state $s_{10}$, and state $s_{10}$ disappears from the strongly fuzzy equilibrium list while state $s_{12}$ joins the weakly fuzzy stable state list. The results show that the fuzziness of JS's preferences is pretty strong, and JS's different FSTs will influence the conflict's development and solutions. Specifically, if JS is more economic-oriented and does not want to cooperate, the conflict will have more chances to stay at state $S_{10}$ (JS stays the status quo, ZJ blocks 
Table 8. The fuzzy stability analysis results.

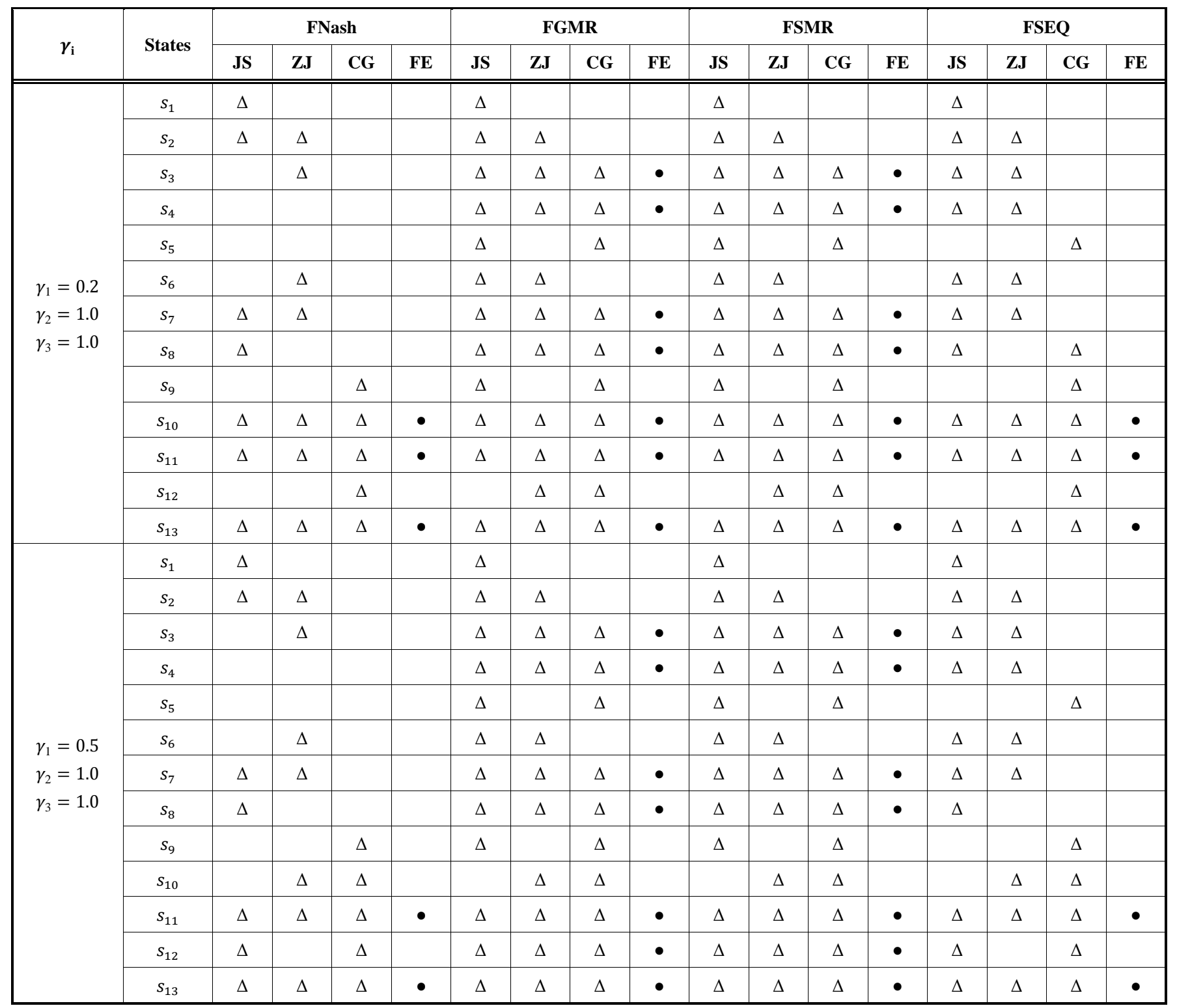

the river, and CG chooses "Punish" policy to coordinate the conflict), where the conflict cannot be solved effectively; if JS is more environmental-oriented and would like to cooperate, state $s_{11}$ (JS reduces the pollution, $\mathrm{ZJ}$ removes the blockage in the river, and CG selects "Punish" policy) will have more opportunities to become an equilibrium which is favorable for all DMs. Notice that although state $s_{13}$ (a distinguishable state when JS chooses option $\mathrm{A}_{3}$ ) is a strongly fuzzy stable state when both $\gamma_{1}=0.2$ and $\gamma_{1}=0.5$, it did not happen in reality, because totally shutting down all the polluting industries is the worst situation for JS, and hence is just a beautiful wish of $\mathrm{ZJ}$ and $\mathrm{CG}$.

\subsubsection{Results Discussion}

In reality, the status quo state of the conflict was state $s_{1}$, from which the conflict was transferred to state $s_{2}$ because ZJ changed his option from "Negotiate" to "Block". After the conflict was intensified by ZJ's blockage of the river, $\mathrm{CG}$ started to use "Punish" instead of "Persuade" policy to coordinate the conflict, hence the conflict developed toward state $s_{10}$. With the pressures from both ZJ and CG, JS decided to reduce its pollution, which resulted in the happening of state $s_{12}$. Finally, ZJ removed the blockage and the conflict was solved and became stable at state $s_{11}$. It can be seen that the above fuzzy stability analysis is consistent with the actual trajectory of the conflict, which verifies the applicability and feasibility of the conflict analysis model with fuzzy preferences.

From the above analysis, one can see that using GMCR, firstly, the revolution of a water resources conflict can be simulated through dynamically tracking the possible moves and countermoves of DMs among different states in the conflict; and secondly, the possible solutions of the conflict can be calculated to provide strategic guidance for DMs in the conflict and other conflict analyzers. 


\section{CONCLUSION}

The fuzzy preference framework of GMCR, which can handle conflicts with both crisp and fuzzy preferences of DMs, is introduced in this paper, and is then applied to model and analyze a water contamination conflict in China. The fuzzy stability analysis results shown in Table 8 demonstrate how a DM's satisficing criteria (FST) can affect the DM's preferences and the final conflict resolutions. Actually, the fuzzy preference framework of GMCR is capable of employing different FSTs of DMs to predict different possible solutions for the conflict. Therefore, this method can be interpreted not only as predictions, but also as answers to "WhatIf?" questions, which is a significant function for both practitioners and researchers to obtain more strategic insights. In addition, a useful technique of fuzzy option prioritization, which can represent both crisp and fuzzy preferences, is used to effectively elicit the fuzzy preferences of JS and the crisp preferences of ZJ and CG. This efficient tool will definitely enhance the applicability of the fuzzy preference framework of GMCR.

Fuzzy stability analysis under the framework of GMCR is a new concept. It might be integrated with other developments within the framework of GMCR, such as attitudes analysis, coalition analysis, and status quo analysis. Calculating various fuzzy stabilities by hand is tedious even for a small-size conflict model, hence designing a suitable decision support system for the fuzzy preference framework of GMCR is an important future project. Additionally, most research in conflict analysis including GMCR assumes symmetrical roles for the DMs, which are not true in some real-world cases. Therefore, one extension for GMCR can be developing a formal procedure within the framework of GMCR to study DM's asymmetric status.

\section{CONFLICT OF INTEREST}

The authors confirm that this article content has no conflict of interest.

\section{ACKNOWLEDGEMENTS}

The authors express their sincere appreciation to the anonymous referees, and the editors for furnishing constructive suggestions.

\section{REFERENCES}

[1] D. M. Kilgour, K. W. Hipel, and L. Fang, "The graph model for conflicts", Automatica, vol. 23, pp. 41-55, 1987.

[2] L. Fang, K. W. Hipel, and D. M. Kilgour, Interactive Decision Making: The Graph Model for Conflict Resolution. John Wiley, Hoboken, 1993.

[3] K. W. Hipel, and A. Obeidi, "Trade versus the environment: Strategic settlement from a systems engineering perspective", Systems Engineering, vol. 8, pp. 211-233, 2005.

[4] K. W. Hipel, L. Fang, and D. M. Kilgour, "Decision support systems in water resources and environmental management", Journal of Hydrologic Engineering, vol. 13, pp. 761-770, 2008.

[5] S. Walker, T. Boutilier, and K. W. Hipel, "Systems management study of a private brownfield renovation", Journal of Urban Planning and Development, vol. 136, pp. 249-260, 2010.

[6] D. M. Kilgour, K. W. Hipel, L. Fang, and X. Peng, "Coalition analysis in group decision support", Group Decision and Negotiation, vol. 10, pp. 159-175, 2001.

[7] K. W. Li, D. M. Kilgour, and K. W. Hipel, "Status quo analysis of the Flathead River conflict", Water Resources Research, vol. 40, 2004.

[8] T. Inohara, K. W. Hipel, and S. Walker, "Conflict analysis approaches for investigating attitudes and misperceptions in the War of 1812", Journal of Systems Science and Systems Engineering, vol. 16, pp. 181-201, 2007.

[9] J. Yu, M. Zhao, and Y. Chen, "Decision makers' attitudes analysis under the framework of GMCR", Soft Science, vol. 29, pp. 140$144,2015$.

[10] J. Yu, K. W. Hipel, D. M. Kilgour and M. Zhao, "Option prioritization for unknown preference", Journal of Systems Science and Systems Engineering, 2015 [Epub ahead of print].

[11] M. A. Bashar, D. M. Kilgour, and K. W. Hipel, "Fuzzy preferences in the graph model for conflict resolution", Fuzzy Systems, vol. 20, pp. 760-770, 2012

[12] K. W. Hipel, D. M. Kilgour, L. Fang, and X. Peng, "The decision support system GMCR in environmental conflict management", Applied Mathematics and Computation, vol. 83, pp. 117-152, 1997.

[13] L. Fang, K. W. Hipel, D. M. Kilgour, and X. Peng, "A decision support system for interactive decision making-Part I: model formulation", Systems, Man, and Cybernetics, Part C: Applications and Reviews, vol. 33, pp. 42-55, 2003.

[14] L. Fang, K. W. Hipel, D. M. Kilgour, and X. Peng, "A decision support system for interactive decision making-Part II: analysis and output interpretation", Systems, Man, and Cybernetics, Part C: Applications and Reviews, vol. 33, pp. 56-66, 2003.

[15] M. A. Bashar, D. M. Kilgour, and K. W. Hipel, "Fuzzy option prioritization for the graph model for conflict resolution", Fuzzy Sets and Systems, vol. 246, pp. 34-48, 2014.

[16] J. Yu, M. Zhao, D. Sun, and J. Dong, "Research on the water pollution conflict between the upstream area and the downstream area in a river basin based on GMCR", Journal of Hydraulic Engineering, vol. 44, pp. 1389-1398, 2013. 